\title{
Utility of Abnormal Head Computed Tomography in Predicting Outcome in Out-of-Hospital Cardiac Arrest Victims
}

\author{
Rebecca Fisher, MD, ${ }^{1}$ Michael J. Bernett, MD, MBA, ${ }^{2}$ Ryan Paternoster, CCRP, ${ }^{3}$ \\ Patrick Karabon, MS, William Devlin, MD, ${ }^{4}$ and Robert Swor, DO ${ }^{1}$
}

\begin{abstract}
Head computed tomography (HCT) is often performed postcardiac arrest to assess for hypoxic-ischemic brain injury. Our primary objective was to assess whether cerebral edema (CE) on early HCT is associated with poor survival and neurologic outcome after out-of-hospital cardiac arrest (OHCA).We included subjects from a prospectively collected database of OHCA adults who received targeted temperature management at two hospitals from July 2009 to July 2018. We included cases if an emergency department (ED) HCT was performed. Patient demographics and cardiac arrest variables were collected. HCT results were abstracted from radiology reports. HCT findings were categorized as no acute disease, evidence of CE, or excluded (bleed, tumor, and stroke). Outcomes were survival to discharge or dichotomized discharge cerebral performance category (CPC) of 1-2 (good neurologic outcome) versus 3-5 (poor neurologic outcome). Univariate and multivariate analyses were performed. There were 425 OHCA, of which 315 had ED HCT with 277 cases included. Patients were predominately male $(65.0 \%)$, average age of 60.9 years and average body mass index of 30.5. Of all cases, 44 (15.9\%) showed CE on computed tomography. Univariate analysis demonstrated that CE was associated with 9.2-fold greater odds of poor outcome (odds ratio [OR]: 9.23; 95\% confidence interval [CI] 1.73-49.2) and 9.1-fold greater odds of death (OR: 9.09, 95\% CI 2.4-33.9). In adjusted analysis, CE was associated with a poor CPC outcome (adjusted odds ratios [AOR]: 14.9, 95\% CI 2.49-88.4), and death (AOR: 13.7, 95\% CI 3.26-57.4). Adjusted survival analysis demonstrated that patients with CE on HCT had 3.6-fold greater hazard of death than those without CE (hazard ratios 3.56, 95\% CI 2.34-5.41). The results identify that $\mathrm{CE}$ on HCTs early in the postarrest period in OHCA patients is strongly associated with poor rates of survival and neurologic outcome. Prospective work is needed to further define the role of early HCT in postarrest neuroprognostication.
\end{abstract}

Keywords: head computed tomography, out-of-hospital cardiac arrest, hypoxic-ischemic brain injury, cerebral edema

\section{Introduction}

$\mathbf{I}^{\mathrm{N}}$ N THE UNITED STATES, out-of-hospital cardiac arrest (OHCA) occurs in an estimated 400,000 patients every year (Ringh et al., 2018). Of these victims, only $22 \%$ will achieve return of spontaneous circulation; a mere $10.5 \%$ will survive to hospital discharge (CARES, 2019). Poor postarrest survival rates are the result of underlying disease that precipitates the initial event, and the combination of initial cardiac and neurologic insult as well as subsequent reperfusion injury (Nolan et al., 2008).
Hypoxic-ischemic brain injury (HBI) is identified as the primary cause of death in patients resuscitated from cardiac arrest (Dragancea et al., 2013). Cerebral edema (CE) is one marker of HBI and is a global insult, without the opportunity to mitigate insult due to collateral vasculature. $\mathrm{HBI}$ is thought to be caused by multiple factors, including $\mathrm{CE}$, which can then result in increased intracranial pressure, herniation, and brain death (Sekhon et al., 2017). Targeted temperature management (TTM) is recommended treatment for minimizing further neurologic injury following a cardiac arrest (Tahsili-Fahadan et al., 2018), with a low-grade quality of evidence (Donnino

\footnotetext{
${ }^{1}$ Department of Emergency Medicine, Beaumont Health System, Royal Oak, Michigan, USA.

${ }^{2}$ Geisinger Medical Center, Danville, Pennsylvania, USA.

${ }^{3}$ Office of Research, Oakland University William Beaumont School of Medicine, Royal Oak, Michigan, USA.

${ }^{4}$ Beaumont Hospital-Troy, Beaumont Health System, Royal Oak, Michigan, USA.
} 
et al., 2015). Despite an extensive body of literature regarding neuroprognostication of postarrest patients, the optimal method of determining prognosis postresuscitation remains elusive. The European Resuscitation Council and European Society of Intensive Care Medicine released updated guidelines in 2015 recommending a multimodal strategy, including clinical examination, electrophysiology studies, biomarkers, including neuron-specific enolase, and imaging (Nolan et al., 2015). These guidelines do not identify variables that may be used in the early postarrest period that reliably determine prognosis.

There are important implications in regard to the timing of prognosticating patient outcomes (neuroprognostication). Early determination of poor prognosis may lead to premature decisions to withdraw further care (Perman et al., 2012). Reliable information regarding prognosis are vital to inform both clinicians and family members. Current guidelines recommend clinicians to wait more than 72 hours after a patient is rewarmed, after receiving TTM with sedation and/or paralytics to determine whether or not critical care should be withdrawn or if further observation is warranted (Callaway et al., 2015). Head computed tomography (HCT) is often used to help clinicians identify intracranial pathology that may have led to a cardiac arrest and to identify injury that may be a result of the arrest. It is often used by clinicians to assess the degree of anoxic injury. Findings of $\mathrm{CE}$ and loss of gray matter to white matter differentiation ratio (GWR) on HCT have been documented to be predictive of poor neurologic outcome after hypoxic brain damage (Yamamura et al., 2013).

This study aimed to evaluate the utility of the emergency department (ED) HCT in predicting neurologic outcome and survival to hospital discharge in OHCA victims successfully resuscitated and subsequently placed on TTM.

\section{Methods}

We performed a secondary analysis of a prospectively collected cardiac arrest database, which included patients who received TTM at Beaumont Royal Oak or Troy Hospitals from July 2009 to July 2018. All patients with an OHCA were included if an HCT was performed at any point during ED course. Cases were excluded if arrest victims did not receive an HCT in the ED, did not receive TTM, were arrested in the hospital setting, or were transferred from outside facilities.

Patient records were reviewed for demographic information as well as cardiac arrest variables. These variables included if the arrest was witnessed and their initial rhythm on cardiac monitoring. HCT results for each patient case were abstracted from the original radiology reports. HCT findings were categorized as normal, abnormal (CE), or other (bleed, tumor, or stroke). Two investigators (M.J.B. and R.F.) reviewed all HCTs identified as having $\mathrm{CE}$ and an equal number of controls (selected based on normal radiology reports) to assess inter-rater reliability. A third author (R.S.) resolved discordant coding determinations by reviewing the original radiology reports.

To assess for neurologic outcome, cerebral performance category (CPC) was assigned by research associates upon hospital discharge and recorded in the registry. CPC is widely used in research as an assessment tool to interpret neurologic status in patients following cardiac arrest incidents (Ajam et al., 2011). A CPC of 1-2 was defined as a good outcome, with CPC 3-5 defined as poor outcome.

\section{Statistical analysis}

Descriptive statistics were run with continuously measured variables displayed in terms of mean/average with standard deviation in parentheses and categorical variables displayed in terms of frequencies with percentages in parentheses. Univariate tables were evaluated using two-sample independent $T$-tests for continuous variables and Chi-squared tests for categorical variables. Odds ratios (OR) with corresponding $95 \%$ confidence intervals (95\% CI) were also generated for categorical variables. A multivariate logistic regression was generated to model a poor neurological outcome. Results were displayed in terms of adjusted odds ratios (AOR) with $95 \% \mathrm{CI}$ and $p$-values.

For a survival analysis, actuarial survival estimates with 95\% CI were generated at 2, 4, 6, and 8 days after computed tomography (CT) scan. Kaplan-Meier curves, stratified by $\mathrm{CE}$ on CT, were generated. Finally, a multivariate Cox proportional hazards regression was run, with results displayed in terms of adjusted hazard ratios (HR) with 95\% CI and $p$-values. The assumptions of proportional hazards were met.

An inter-rater reliability analysis to compare the agreement between the two investigators in terms of interpretations of HCT radiology reports was formally assessed using Cohen's Kappa.

To avoid overfitting in the multivariate models, Firth Penalized Likelihood was used throughout this study. This study received approval through the Beaumont Health Institutional Review Board. Any $p$-value $<0.05$ indicates a statistically significant association. All analysis was performed in SAS 9.4 (SAS Institute, Inc., Cary, NC, USA).

\section{Results}

During the study period, there were $425 \mathrm{OHCA}$, of which 315 cases had ED HCTs performed and 277 were included for initial analysis (see Fig. 1 for consort diagram). Patients who received ED HCT were less likely to have been a witnessed arrest or have an initial shockable rhythm, and more likely to arrest in a public location (Table 1). Current protocol at the study hospitals does not require an HCT for OHCA. Subject demographics that met inclusion criteria are compared to those who did not receive an HCT and are reported in Table 2. Of the included patients, 254 cases were included in the final survival analysis, with exclusions due to lack of temporal data from HCT to final outcome. Patients were predominately male $(65.0 \%)$, with an average age of 60.9 years and an average body mass index of 30.5. HCT was performed relatively early in the subject's course in the ED (median 90 [62-131] minutes).

Of all cases, 44 (15.9\%) showed CE on ED HCT. Patients with $\mathrm{CE}$ on HCT less often sustained a witnessed arrest (11.0\% vs. $28.6 \%, \mathrm{OR}=0.31,95 \%$ CI $0.16-0.60)$, less frequently had an initial shockable rhythm (9.1\% vs. $19.1 \%$, $\mathrm{OR}=0.44$, 95\% CI 0.20-0.99), and surprisingly, were younger ( 47.0 vs. 63.5 years, $p<0.001)$. Univariate analysis demonstrated that $\mathrm{CE}$ was associated with 9.2-fold greater odds of poor outcome (OR: 9.23; 95\% CI 1.73-49.2) and 9.1-fold greater odds of death (OR: 9.09: 95\% CI 2.43-33.9) (Table 3$)$. One patient had a good outcome $(2.3 \%, 95 \%$ CI 0.0-6.7. In the adjusted analysis, CE was associated with 14.9-fold greater odds of poor CPC outcome (AOR: 14.9, 95\% CI 2.49-88.4) and 13.7-fold greater odds of death 
FIG. 1. Consort diagram of included patients. CT, computed tomography; ED, emergency department.



(AOR: 13.7, 95\% CI 3.26-57.4) (Table 4). The adjusted survival analysis demonstrated that patients with $\mathrm{CE}$ on HCT had 3.6-fold greater hazard of death than those without edema on HCT (HR: 3.56: 95\% CI 2.34-5.41) (Table 5 and Fig. 2). Inter-rater reliability demonstrated excellent agreement between the two reviewers for CE on HCT $(\kappa=0.86)$.

\section{Discussion}

In this study, we identified that the presence of CE on HCT in the ED phase of postarrest care was infrequent, but associated with a marked decrease in patients' survival to hospital discharge and predictive of poor neurologic outcome in survival (CPC 3-5). Of the patients identified with $\mathrm{CE}$ on HCT, only one survived with a good neurologic outcome. In our population, $\mathrm{CE}$ was more likely to be found in arrests with a lower chance of survival, such as in older patients, unwitnessed arrests, and patients with a nonshockable rhythm.

\begin{tabular}{lc}
\multicolumn{2}{c}{ TABle 1. Subject Demographics } \\
\hline \multicolumn{1}{c}{ Patients } \\
Demographics & $(\mathrm{N}=277)$ \\
\hline Age (mean in years, SD) & $60.9(18.2)$ \\
Gender $(N, \%$ male) & $180(68.2 \%)$ \\
BMI (mean, SD) & $30.5(8.2)$ \\
Survival to hospital discharge & $83(30 \%)$ \\
CPC on discharge, $n(\%)$ & \\
$\quad$ Poor CPC (3-5) & $220(79.4)$ \\
$\quad$ Good CPC (1-5) & $57(20.6)$ \\
CE on CT, $n(\%)$ & $44(15.9)$ \\
Witnessed cardiac arrest, $n(\%)$ & $200(72.2)$ \\
Initial rhythm: ventricular fibrillation, $n(\%)$ & $88(31.8)$
\end{tabular}

BMI, body mass index; CE, cerebral edema; CPC, cerebral performance category; CT, computed tomography; SD, standard deviation.
To improve poor survival rates of postcardiac arrest victims, clinicians need to be able to quickly identify potentially lethal clinical entities that are present and act to mitigate further injury. HCT as part of the early postarrest care of patients may be important to identify potential cause of cardiac arrest (e.g., intracranial hemorrhage), injury related to the event (e.g., traumatic intracranial injury), or other pathology (e.g., tumor and ischemic stroke) that may result in cardiac arrest. Identification of CE may also identify a clinical entity amenable to treatment or be an early predictor of poor outcome. Traditional measures to treat CE after posttraumatic injury or tumor such as osmotic agents, steroids, or interventional intracranial pressure monitoring have not been validated in postcardiac arrest care (Nolan et al., 2008). The value of early HCT for treatment is unclear. Our data add to

Table 2. Comparison of Patients Who Did or Did Not Have Computed Tomography Performed

\begin{tabular}{lcc}
\hline & No ED CT & ED CT \\
\hline$N$ & 109 & 310 \\
Gender (\%) & & \\
$\quad \%$ Male & 61.5 & 66.0 \\
$\quad \%$ Female & 38.5 & 34.0 \\
Race (\%) & & \\
$\quad$ Caucasian & 75.7 & 78.7 \\
$\quad$ African American & 14.0 & 14.5 \\
$\quad$ Other & 10.3 & 3.9 \\
Age (average years) & 62.8 & 61.2 \\
BMI & 31.8 & 30.4 \\
Witnessed arrest (\%) & 78.2 & 70.5 \\
Shockable rhythm (\%) & 46.4 & 32.7 \\
Survival to discharge (\%) & 38.5 & 32.4 \\
Survival with good outcome (\%) & 25.2 & 20.5 \\
\hline
\end{tabular}

ED, emergency department. 
Table 3. Univariate Analysis of Computed Tomography and Outcome

\begin{tabular}{|c|c|c|c|c|}
\hline & Poor CPC $(\mathrm{n}=200)$ & Good CPC $(\mathrm{n}=57)$ & OR $(95 \% C I)$ & $\mathrm{p}$ \\
\hline Age & $62.52(18.16)$ & $54.54(16.85)$ & N/A & 0.0030 \\
\hline BMI & $31.05(8.53)$ & $28.51(16.29)$ & N/A & 0.0135 \\
\hline \multicolumn{5}{|l|}{$\mathrm{CE}$ on $\mathrm{CT}$} \\
\hline Yes & $43(97.73 \%)$ & $1(2.77 \%)$ & $9.23(1.73-49.2)$ & \multirow[t]{2}{*}{0.0092} \\
\hline No & $177(75.97 \%)$ & $56(24.03 \%)$ & Reference Group & \\
\hline \multicolumn{5}{|c|}{ Witnessed cardiac arrest } \\
\hline Yes & $157(78.50 \%)$ & $43(21.50 \%)$ & $0.83(0.43-1.61)$ & \multirow{2}{*}{0.5752} \\
\hline No & $63(81.82 \%)$ & $14(18.18 \%)$ & Reference Group & \\
\hline \multicolumn{5}{|c|}{ Initial rhythm: Vfib } \\
\hline Yes & $48(54.55 \%)$ & $40(45.45 \%)$ & $0.12(0.06-0.23)$ & \multirow{2}{*}{$<0.0001$} \\
\hline No & $172(91.01 \%)$ & $17(8.99 \%)$ & Reference Group & \\
\hline \multicolumn{5}{|c|}{ Gender of patient } \\
\hline Female & $76(78.35 \%)$ & $21(21.65 \%)$ & $0.90(0.49-1.64)$ & \multirow{2}{*}{0.7288} \\
\hline Male & $144(80.00 \%)$ & $36(20.00 \%)$ & Reference Group & \\
\hline
\end{tabular}

the body of knowledge that demonstrates CE on early HCT is associated with a poor outcome, given that in our patient population, only one patient with CE on HCT survived with a favorable outcome.

We found that when obtained during a patient's initial workup after ROSC in the ED, HCT was useful as a neuroprognostication tool and a predictor of survival outcomes. Moseby-Knappe et al. performed a similar retrospective study, and of 91 patients with CE on HCT, only 4 survived with CPC scores of 1-2. They believe two of those four patients may have been incorrectly categorized as having $\mathrm{CE}$ on HCT by the radiologist after data review (MosebyKnappe et al., 2017). Cristia et al. (2014) categorized patients as no edema, mild edema, and severe edema, and found that the 11 patients they categorized as having severe edema on HCT had a $100 \%$ mortality rate.

Although we did not quantify CE in our study, numerous studies have made efforts toward quantifying CE using gray matter to white matter attenuation ratios (GWR), as increasing CE decreases the GWR. Torbey et al. compared the GWR on HCT of 25 comatose patients after cardiac arrest to 25 noncardiac arrest patients, whose HCTs were determined

Table 4. Multivariate Model and Poor Neurological Outcome (CPC Score 3, 4, AND 5)

\begin{tabular}{lcc}
\hline & AOR $(95 \%$ CI $)$ & $\mathrm{p}$ \\
\hline Age & $1.04(1.02-1.06)$ & 0.0001 \\
BMI & $1.02(0.98-1.07)$ & 0.2856 \\
CE on CT & $14.9(2.49-88.4)$ & 0.0003 \\
$\quad$ Yes & Reference group & \\
$\quad$ No & $1.20(0.54-2.65)$ & \\
Witnessed cardiac & arrest & 0.6565 \\
Yes & Reference group & \\
No & $0.12(0.06-0.25)$ & \\
Initial rhythm: ventricular fibrillation & \\
$\quad$ Yes & Reference group & \\
No & & \\
Gender of patient & $0.57(0.28-1.17)$ & 0.0001 \\
Male & Reference group & \\
Female & & \\
\hline
\end{tabular}

AOR, adjusted odds ratio. to be normal. In their study population, the HCT was performed within the first 48 hours of admission. The GWR was significantly lower in the comatose patients versus the control group, with a $100 \%$ mortality rate if the GWR was $<1.18$ at the level of the basal ganglia (Torbey et al., 2001). These data were supported by Gentsch et al. (2015), who found $100 \%$ specificity and $44.3 \%$ sensitivity with a GWR cutoff of 1.16 in the basal ganglia in their patient population, and Scheel et al. (2013) with $100 \%$ specificity and $38 \%$ sensitivity. Similar to our time to HCT, BK Lee et al. (2016) found the GWR was significantly associated with patient's neurological outcome at a median time to HCT of 1 hour. Metter et al. (2011) approximated GWR in the basal ganglia and cerebrum, and found that the average GWR was higher in survivors of cardiac arrest as well. Comparable studies by Kim et al. (2019) in efforts to determine an appropriate GWR cutoff with $100 \%$ specificity for poor neurological outcome after cardiac arrest have shown similar results.

Our study has limitations. The total ischemic time of cardiac arrest was not recorded in this study, and thus we cannot exclude the relationship of length of resuscitation and subsequent development of CE.

Table 5. COX Proportional Hazard RATIO FOR DEATH

\begin{tabular}{lcc}
\hline & $H R(95 \%$ CI $)$ & $\mathrm{p}$ \\
\hline CE on CT & $3.56(2.34-5.41)$ & $<0.0001$ \\
Yes & Reference group & \\
No & $1.02(1.01-1.03)$ & 0.0001 \\
Age & $1.02(0.99-1.03)$ & 0.2610 \\
BMI & $0.92(0.66-1.28)$ & 0.6010 \\
Witnessed cardiac & arrest \\
Yes & Reference group & \\
No & $0.31(0.21-0.46)$ & $<0.0001$ \\
Initial rhythm: ventricular fibrillation & \\
Yes & Reference group & \\
No & & \\
Gender of patient & $0.85(0.62-1.16)$ & 0.2986 \\
Female & Reference group & \\
Male &
\end{tabular}

HR, hazard ratios. 
FIG. 2. Kaplan-Meier analysis for survival.



Furthermore, while we were able to ascertain that HCTs were performed during the course of the ED stay, we did not factor into our analysis the time of cardiac arrest until time of HCT. Our average time to HCT was 1 hour and 30 minutes (interquartile range: 1:02, 2:11). A longer time to HCT may have allowed more time for the development of CE. Streitberger et al. evaluated patients receiving two HCTs, one within 24 hours with a repeat more than 24 hours after cardiac arrest. Their study showed that HCTs performed greater than 24 hours after cardiac arrest had an increased sensitivity and they concluded HCTs for neuroprognostication should occur at least 24 hours after achieving ROSC (Streitberger et al., 2019). Scheel et al. (2013) also showed decreasing GWR on HCT in the days following cardiac arrest, and state that the sensitivity of HCT increases $>24$ hours after admission. Wang et al. (2018) did not find a significant difference between GWR and poor outcomes when HCT was performed early, but did not rule out that a later CT could be used in predicting neurological outcome. CT after 24 hours may be more useful for finalizing a determination of poor outcome at the expense of having early important data to inform decision making of clinicians and families.

Our results may not be directly applicable to all patients who present to the ED postcardiac arrest. Patients who received an ED HCT were less likely witnessed arrests or initially in ventricular fibrillation, and were a study population at higher risk for adverse outcomes. In the initial cohort, $26 \%$ of patients did not receive an ED HCT (Table 2). We used CPC as an outcome variable for this study, which has previously been identified as an imperfect measure of cardiac arrest outcome (Becker et al., 2011). Concerns with CPC include the lack of discrimination between scores, along with overestimation of the patient's function (Haywood et al., 2018). However, there has been research into the correlation between $\mathrm{CPC}$ at hospital discharge and 1-, 6-, and 12-month survival of patients who received TTM after OHCA, which shows CPC 1-3 have the highest rates of long-term survival (Hsu et al., 2014).

We identified one patient who had CE on initial CT and who had a good outcome. Our data identify that the presences of CE on CT is associated with markedly worse odds of a good outcome, but is not uniformly predictive of poor survival. The upper bound of the $95 \%$ CI of the false positive identifies that up to $6.7 \%$ may have $\mathrm{CE}$ yet have a good outcome, so it may not be used as a definitive test. We believe this places early CT in the context of most other tests, not uniformly definitive of a poor outcome, but it is important data, which may be used in context of other clinical and patient-level variables in the determination of further care.

\section{Conclusion}

In the population of patients resuscitated from OHCA, findings of $\mathrm{CE}$ on HCT were associated with low rates of survival and, among survivors, poor neurologic outcomes. ED HCT is a useful adjunct in determining prognosis for patients who are resuscitated from OHCA, and further research into this may help improve neuroprognostication algorithms to reduce the delivery of nonbeneficial care and allow clinicians and families to focus on realistic goals of care.

\section{Author Disclosure Statement}

No competing financial interests exist.

\section{Funding Information}

No funding was received.

\section{References}

Ajam K, Gold LS, Beck SS, Damon S, Phelps R, Rea TD. Reliability of the Cerebral Performance Category to classify neurological status among survivors of ventricular fibrillation 
arrest: A cohort study. Scand J Trauma Resusc Emerg Med 2011;19:38.

Becker LB, Aufderheide TP, Geocadin RG, et al. Primary outcomes for resuscitation science studies. Circulation 2011; 124:2158-2177.

Callaway CW, Donnino MW, Fink EL, et al. Part 8: Postcardiac arrest care. Circulation 2015;132(18 suppl 2): S465-S482.

CARES. 2019 Hospital Survival Report (Accessed on August 11, 2020 at mycares.net).

Cristia C, Ho ML, Levy S, et al. The association between a quantitative computed tomography (CT) measurement of cerebral edema and outcomes in post-cardiac arrest-A validation study. Resuscitation 2014;85:1348-1353.

Donnino MW, Andersen LW, Berg KM, et al. Temperature management after cardiac arrest. Circulation 2015;132:24482456.

Dragancea I, Rundgren M, Englund E, et al. The influence of induced hypothermia and delayed prognostication on the mode of death after cardiac arrest. Resuscitation 2013;84: 337-342.

Gentsch A, Storm C, Leithner C, et al. Outcome prediction in patients after cardiac arrest: A simplified method for determination of gray-white matter ratio in cranial computed tomography. Clin Neuroradiol 2015;25:49-54.

Haywood K, Whitehead L, Nadkarni VM, et al. COSCA (Core Outcome Set for Cardiac Arrest) in adults: An advisory statement from the International Liaison Committee on Resuscitation. Circulation 2018;137:e783-e801.

Hsu CH, Li J, Cinousis MJ, et al. Cerebral performance category at hospital discharge predicts long-term survival of cardiac arrest survivors receiving targeted temperature management. Crit Care Med 2014;42:2575-2581

Kim JH, Kim MJ, You JS, et al. Multimodal approach for neurologic prognostication of out-of-hospital cardiac arrest patients undergoing targeted temperature management. Resuscitation 2019;134:33-40.

Lee BK, Kim WY, Shin J, et al. Prognostic value of gray matter to white matter ratio in hypoxic and non-hypoxic cardiac arrest with non-cardiac etiology. Am J Emerg Med 2016;34: 1583-1588.

Metter RB, Rittenberger JC, Guyette FX, Callway CW. Association between a quantitative CT scan measure of brain edema and outcome after cardiac arrest. Resuscitation 2011; 82:1180-1185.

Moseby-Knappe M, Pellis T, Dragancea I, et al. Head computed tomography for prognostication of poor outcome in comatose patients after cardiac arrest and targeted temperature management. Resuscitation 2017;119:89-94.
Nolan JP, Neumar RW, Adrie C, et al. Post-cardiac arrest syndrome: Epidemiology, pathophysiology, treatment, and prognostication A Scientific Statement from the International Liaison Committee on Resuscitation; the American Heart Association Emergency Cardiovascular Care Committee; the Counc. Resuscitation 2008;79:350-379.

Nolan JP, Soar J, Cariou A, et al. European Resuscitation Council and European Society of Intensive Care Medicine Guidelines for post-resuscitation care 2015. Resuscitation 2015;95:202-222.

Perman SM, Kirkpatrick JN, Reitsma AM, et al. Timing of neuroprognostication in postcardiac arrest therapeutic hypothermia. Crit Care Med 2012;40:719-724.

Ringh M, Hollenberg J, Palsgaard-Moeller T, et al. The challenges and possibilities of public access defibrillation. J Intern Med 2018;283:238-256.

Scheel M, Storm C, Gentsch A, et al. The prognostic value of gray-white-matter ratio in cardiac arrest patients treated with hypothermia. Scand J Trauma Resus Emerg Med 2013;21:23.

Sekhon MS, Ainslie PN, Griesdale DE. Clinical pathophysiology of hypoxic ischemic brain injury after cardiac arrest: A "two-hit" model. Crit Care 2017;21:90.

Streitberger KJ, Endsich C, Ploner CJ, et al. Timing of brain computed tomography and accuracy of outcome prediction after cardiac arrest. Resuscitation 2019;145:8-14.

Tahsili-Fahadan P, Farrokh S, Geocadin RG. Hypothermia and brain inflammation after cardiac arrest. Brain Circ 2018;4:1-13.

Torbey MT, Selim J, Knorr C, et al. Quantitative analysis of the loss of distinction between gray and white matter in comatose patients after cardiac arrest. Stroke 2001;31:2163-2167.

Wang GN, Chen XF, Lv JR et al. The prognostic value of graywhite matter ratio on brain computed tomography in adult comatose cardiac arrest survivors. J Chin Med Assoc 2018; 81:599-604.

Yamamura H, Kaga S, Kaneda K, et al. Head Computed Tomographic measurement as an early predictor of outcome in hypoxic-ischemic brain damage patients treated with hypothermia therapy. Scand J Trauma Resusc Emerg Med 2013; 21:37.

Address correspondence to: Rebecca Fisher, MD

Department of Emergency Medicine William Beaumont Hospital 3601 W. Thirteen Mile Road Royal Oak, MI 48073

USA

E-mail: rebecca.fisher@beaumont.org 